\title{
Quantitative coronary CT angiography: absolute lumen sizing rather than \% stenosis predicts hemodynamically relevant stenosis
}

\author{
Fabian Plank $^{1,2}$ • Philipp Burghard ${ }^{1}$ - Guy Friedrich ${ }^{2}$ • Wolfgang Dichtt ${ }^{2}$ - Agnes Mayr ${ }^{1}$ • \\ Andrea Klauser ${ }^{1} \cdot$ Florian Wolf $^{3} \cdot$ Gudrun Feuchtner $^{1}$
}

Received: 29 October 2015 / Accepted: 18 January 2016 / Published online: 10 February 2016

(C) The Author(s) 2016. This article is published with open access at Springerlink.com

\begin{abstract}
Objective To identify the most accurate quantitative coronary stenosis parameter by CTA for prediction of functional significant coronary stenosis resulting in coronary revascularization.

Methods 160 consecutive patients were prospectively examined with CTA. Proximal coronary stenosis was quantified by minimal lumen area (MLA) and minimal lumen diameter (MLD), \%area and \%diameter stenosis. Lesion length (LL) was measured. The reference standard was invasive coronary angiography (ICA) $(>70 \%$ stenosis, FFR $<0.8)$.

Results 210 coronary segments were included (59\% positive). MLA of $\leq 1.8 \mathrm{~mm}^{2}$ was identified as the optimal cutoff ( $\mathrm{c}=0.97, \mathrm{p}<0.001 ; 95 \%$ CI $0.94-0.99)$ (sensitivity $90.9 \%$, specificity $89.3 \%$ ) for prediction of functionalrelevant stenosis (for MLA $>2.1 \mathrm{~mm}^{2}$ sensitivity was $100 \%)$. The optimal cut-off for MLD was $1.2 \mathrm{~mm}$ ( $\mathrm{c}=0.92$; $\mathrm{p}<0.001 ; 95 \%$ CI $0.88-95$ ) (sensitivity 90.9 , specificity 85.2 ) while \%area and \%diameter stenosis were less accurate ( $\mathrm{c}=0.89 ; 95 \%$ CI $0.84-93, \mathrm{c}=0.87 ; 95 \%$ CI $0.82-92$, respectively, with thresholds at $73 \%$ and $61 \%$ stenosis). Accuracy for LL was $\mathrm{c}=0.74$ (95\% CI 0.67-81), and for LL/MLA and LL/MLD ratio $\mathrm{c}=0.90$ and $\mathrm{c}=0.84$.
\end{abstract}

Gudrun Feuchtner

gudrun.feuchtner@gmail.com

1 Department of Radiology, Innsbruck Medical University, Anichstrasse 35, A-6020 Innsbruck, Austria

2 Department of Internal Medicine III - Cardiology, Innsbruck Medical University, Innsbruck, Austria

3 Department of Cardiovascular and Interventional Radiology, Vienna Medical University, Vienna, Austria
Conclusions MLA $\leq 1.8 \mathrm{~mm}^{2}$ and MLD $\leq 1.2 \mathrm{~mm}$ are the most accurate cut-offs for prediction of haemodynamically significant stenosis by ICA, with a higher accuracy than relative $\%$ stenosis.

Key Points

- Quantitative coronary CT-angiography is accurate for prediction of functional relevant stenosis.

- Absolute lumen area and diameter rather than \%stenosis predict functional relevance.

- Lumen area $<1.8 \mathrm{~mm}^{2}$ and diameter $<1.2 \mathrm{~mm}$ are the most accurate cut-offs.

- Quantitative parameters are helpful for decision-making in terms of patient management.

Keywords Coronary artery disease $\cdot$ Atherosclerosis . Computed tomography $(\mathrm{CT}) \cdot$ Stenosis quantification . Coronary CT angiography
Abbreviations
CTA Computed tomography angiography
ICA Invasive coronary angiography
MLA Minimal lumen area
MLD Minimal lumen diameter

\section{Introduction}

The rate of invasive coronary angiography (ICA) performed worldwide without a consecutive coronary revascularization procedure is high at around $40 \%$ [1]. Anatomical assessment of coronary arteries by computed tomography angiography (CTA) is a powerful noninvasive tool for patients with intermediate risk of coronary artery disease (CAD) for ruling out significant coronary stenosis $[2,3]$ and to detect coronary 
atherosclerosis, as most recently revealed by the prospective randomized PROMISE trial [2].

Thus, coronary CTA acts as an excellent gatekeeper prior to ICA. While CTA has an excellent negative predictive value of $97 \%$, its positive predictive value is moderate [3]. In clinical practice, visual subjective grading of coronary stenosis is performed in order to define further patient management. In patients with mild $<50 \%$ stenosis by CTA, risk factor modification is recommended. and in those with intermediate 50$70 \%$ stenosis myocardial perfusion exams, respectively. In patients with severe stenosis by CTA, a diagnostic invasive angiography is suggested for further evaluation. This classification is subjective, observer-dependent and influenced by a variety of factors such as individual reading skill level. Quantitative coronary vessel and luminal stenosis parameters by CTA including absolute cross-sectional vessel area, diameter and relative \%area and \%diameter stenosis, correlate well with intravascular ultrasound (IVUS) [4], and better with IVUS than ICA [5].

However, a few studies [6,7] have investigated quantitative CTA for prediction of functional significance of a coronary lesion; they enrolled fewer than 100 patients each [6,7]. The accurate assessment of a lesion's functional significance (i.e. 'flow-limiting' lesion) is of paramount importance in defining further patient management, because only flow-limiting lesions with a fractional flow reserve (FFR) $<0.8$ benefit from consecutive coronary revascularization procedures such as percutaneous coronary intervention (PCI) or bypass graft surgery in terms of outcome, according to the FAME trial [8].

Therefore, the purpose of this study was to assess quantitative CTA parameters in a consecutive large patient cohort. We measured absolute parameters (minimal lumen area (MLA) and diameter (MLD)) and relative \% of luminal area and diameter stenosis as well as lesion length by CTA for prediction of functional significant (i.e. flowlimiting) coronary stenosis by ICA requiring coronary revascularization.

\section{Material and methods}

\section{Study design}

Consecutive patients referred for coronary CT angiography for clinical indications [9] between 2005 and 2015 were included into our local ethics committee-approved study database. Of the entire study cohort (2,587 patients screened), 160 patients were retrospectively selected. CTA was compared with ICA including quantitative stenosis and FFR measurement.

\section{Inclusion criteria}

(1) Proximal and mid RCA, LAD and proximal CX (segments $1,2,5,6,7$ and 11 according to the 17-segments AHA model) with a vessel area of $\geq 5 \mathrm{~mm}^{2}$ were included. (2) Visually suspected 'high-grade' stenosis on CTA during routine clinical readouts (estimated $>50 \%$ or $>70 \%$ pending on the reading radiologist). (3) ICA performed within a maximum of 3 weeks.

Follow-up was performed via phone interview and hospital chart results. ICA results (stenosis and FFR), previous ischaemia test (single-photon emission $\mathrm{CT}$ ) and coronary revascularization procedure data either via PCI or coronary artery bypass grafting were collected.

\section{Exclusion criteria}

Previous PCI, myocardial infarction or coronary artery bypass grafting, renal dysfunction (serum glomerular filtration rate (GFR) $<45 \mathrm{ml} / \mathrm{min} / 1.73 \mathrm{~m}^{2}$ ) and pregnancy.

\section{Computed tomography (CT) angiography (CTA)}

Before December 2009, 64-slice CT (75 patients) was utilized, and subsequently 128 -slice dual source CT ( 85 patients). First, a non-contrast-enhanced calcium score (CCS) scan (detector collimation $64 \times 1.5 \mathrm{~mm}, 120 \mathrm{kV}$, ECG-gating, slice thickness $3 \mathrm{~mm}$, medium smooth kernel B35f) was performed and the Agatston Score was calculated [10].

Thereafter, coronary CTA was performed using either a 128-DSCT (Somatom Definition FLASH, Siemens, Erlangen, Germany) or a 64-slice CT (Somatom Sensation 64, Siemens) scanner: detector collimation $2 \times 64 \times 0.6 \mathrm{~mm}$ with a z-flying spot or $64 \times 0.6 \mathrm{~mm}$, rotation time $0.28 \mathrm{~s}$ or $0.33 \mathrm{~s}$, respectively.

For 128-DSCT, prospective ECG-triggering was applied for regular a heart rate $(\mathrm{HR})<65$ beats/min with (1) either high-pitch-spiral-mode, pitch 3.4 , if HR was $<60$ beats/min; or (2) axial-sequential with diastolic padding window if $\mathrm{HR}$ was $60-65$ beats $/ \mathrm{min}$ or systolic if HR was $>65$ beats $/ \mathrm{min}$ ). Retrospective ECG-gating was used in patients with irregular HR. For 64-slice CT, retrospective ECG-gating was applied. Tube voltage was $100 \mathrm{kV}$ in patients with a body mass index (BMI) $<26 \mathrm{~kg} / \mathrm{m}^{2}$ and $120 \mathrm{kV}$ with a BMI $>26 \mathrm{~kg} / \mathrm{m}^{2}$.

A contrast agent with $370 \mathrm{mg} / \mathrm{ml}$ iodine concentration (Iopromide, Ultravist $370^{\mathrm{TM}}$, Bayer Schering, Berlin, Germany) was injected intravenously (flow $4-6 \mathrm{ml} / \mathrm{s}$ followed by 40 -cc saline bolus), triggered into arterial phase (bolus tracking, 100 Hounsfield units (HU) threshold, ascending aorta). Contrast volume varied between 65 and $120 \mathrm{cc}$ according to the individual patient's body weight and scan time.

Axial images were reconstructed $(0.75-\mathrm{mm}$ slice width; increment 0.4 ; medium-smooth kernel B26f) and coronary 
arteries were evaluated using interactive oblique multiplanar reformations (MPR) and curved MPR on a per-segment-base (AHA-modified 17-segment classification) [11].

\section{Coronary stenosis quantification by CTA}

The proximal target lesion was identified on curved MPR and interactive oblique MPR angulations. Stenosis quantification was performed with two different methods:

On interactive oblique MPRs, the cross-sectional lumen area of the target lesion was obtained from perpendicular angulations of the vessel in two planes. 'Zooming' was avoided.

An experienced observer with $>3$ years of experience performed the following absolute quantitative vessel parameter with fixed $\mathrm{C} / \mathrm{W}$ settings, which were used for primary data analysis:

- Minimal lumen area (MLA) of the target lesion was traced with a circumferential region of interest (ROI) adjusted to the circular or non-circular shape of the outer vessel lumen border.

- Minimal lumen diameter (MLD) was measured with a digital caliper. The closest normal proximal crosssectional vessel area and diameter were measured with a ROI and a digital caliper, respectively. If the proximal segment was not appropriate (e.g. due to a ostial lesion) the closest distal reference segment was chosen.

- Relative area and \%diameter stenosis of the lesion as compared to the proximal reference vessel were calculated

- Lesion length was measured.

- The lesion was scored for the presence calcifying plaque, defined as being positive on Agatston calcium scoring (>130 HU) and hyperdense on CTA.

Automated $c M P R$ (SyngoVia ${ }^{\mathrm{TM}}$, Siemens) were utilized for stenosis quantification (MLA, MLD, relative area and diameter stenosis) of the same lesion by another independent observer (6 months of training with a level III reader in consensus). This software automatically extracts the vessel along a centerline and performs automated contour-tracing and stenosis quantification, while only the lesion must be identified by the observer. The MLA was taken from an orthogonal cross-sectional slice along the vessel centre-line. Manual adjustments to automated contour tracing results were made if necessary. C/W settings were fixed for each individual patient during the analysis.

All readers were blinded to invasive coronary angiography (ICA) results.

\section{Invasive coronary angiography (ICA)}

Invasive coronary angiography was performed via a 7-F femoral access using the Judkins technique on a fluoroscopy unit
(Axiom $^{\mathrm{TM}}$, Siemens Medical Systems). Different standard projections (LAO, RAO) were used. FFR was measured in lesions above $50 \%$ stenosis. High-grade (significant) stenosis was defined as having an FFR lower than $<0.8$.

\section{Outcome measures}

Haemodynamically significant (flow-limiting) coronary stenosis (ICA-positive group) was defined as either an invasive FFR of $\leq 0.8$ by ICA or $>70 \%$ by ICA and a previous positive myocardial ischaemia test, followed by a revascularization procedure.

\section{Statistical analysis}

Statistical analysis was performed using SSPSTM software (V17.0, SPSS Inc., Chicago, IL, USA) and Medical (V12.5, Medical Software, Belgium).

Quantitative variables are expressed as means \pm standard deviations (SD); categorical variables are presented as absolute values and percentages. A $p$-value of less than 0.05 was considered statistically significant. Differences between parametric data were tested with the independent t-test and nominal data with the Chi-square test.

Receiver operating curve (ROC) analysis was performed using a step-wise model at 0.1 decimal increments for quantitative CTA stenosis parameters (MLA, MLD, relative area and diameter stenosis, LL, LL/MLA and LL/MLD ratio), in order to identify the optimal threshold. The area under the curve (AUC) was calculated (c-statistic) and the corresponding $95 \%$ confidence intervals (CIs). The combined interreader and inter-post-processing technique correlation (between the two observers applying manual interactive MPR and automated cMPR measurements) was tested using the paired samples t-test for differences, and with paired samples correlation.

\section{Results}

A total of 160 patients were enrolled with 210 proximal vessel segments. Seventy-two (45\%) patients with 124 (59\%) vessel segments were positive for high-grade stenosis by ICA. Table 1 shows the study cohort characteristics. Table 2 shows the quantitative stenosis parameters. Left main (LM) disease prevalence was $8 / 210(3.8 \%)$. Prevalence of calcification at the lesion site and total coronary calcium score (CCS, mean $511.0 \pm 605$ Agatston Units) (Table 1) were not different between the two groups.

Figure 1 shows the ROC analysis, which revealed a higher accuracy of MLA and MLD (c=0.97, $95 \%$ CI 0.94-0.99 and $\mathrm{c}=0.92,95 \%$ CI 0.88-95; panel A) by CTA as compared to relative \%area and diameter stenosis $(\mathrm{c}=0.89$ and $\mathrm{c}=0.87$, panel 
Table 1 Study population

\begin{tabular}{lllc}
\hline & $\begin{array}{l}\mathrm{ICA}- \\
\mathrm{n}=84\end{array}$ & $\begin{array}{l}\mathrm{ICA}+ \\
\mathrm{n}=72\end{array}$ & $\mathrm{p}$-value \\
\hline Age, y & $66.2 \pm 9.7$ & $65.4 \pm 9.3$ & 0.19 \\
Females & $71(84.5 \%)$ & $15(20.8 \%)$ & $<0.0001 *$ \\
BMI $\left(\mathrm{kg} / \mathrm{cm}^{2}\right)$ & $26.48 \pm 4.6$ & $27.27 \pm 5.7$ & 0.37 \\
Smoking & $31(36.9 \%)$ & $31(43 \%)$ & 0.915 \\
Hypertension & $53(63.1 \%)$ & $57(79.2)$ & 0.457 \\
Dyslipidaemia & $48(57.1 \%)$ & $53(73.6 \%)$ & 0.343 \\
Family history & $30(35.7 \%)$ & $31(43.1 \%)$ & 0.663 \\
Diabetes & $16(19 \%)$ & $7(9.7 \%)$ & 0.196 \\
Calcium score (mean Agatston Units $\pm \mathrm{SD})$ & $458 \pm 572$ & $600 \pm 648$ & 0.149 \\
\hline
\end{tabular}

$I C A$ invasive coronary angiography, $B M I$ body mass index

B). The corresponding bar-chart showed a significant lower minimal lumen area $(\mathrm{p}<0.001)$ in patients with flow-limiting functional significant stenosis (ICA positive group) (Fig. 2).

\section{Absolute quantification of stenosis (MLA and MLD)}

A MLA of $\leq 1.8 \mathrm{~mm}^{2}$ was identified as the most accurate cutoff value for haemodynamically significant stenosis by ICA (sensitivity $90.9 \%$, specificity $89.3 \%$ ). Table 3 presents the stepwise ROC results of MLA. All positive lesions had a MLA of $2.1 \mathrm{~mm}^{2}$ or less (sensitivity $100 \%$ ). Table 4 shows step-wise ROC results for MLD, revealing a threshold of $\leq 1.2 \mathrm{~mm}$ as being most accurate.

Box-Plot (Fig. 3) shows a significantly lower MLA in haemodynamically significant stenosis as compared to negatives on ICA $\left(1.34 \mathrm{~mm}^{2}\right.$ vs. $\left.2.61 \mathrm{~mm}^{2} ; \mathrm{p}<0.001\right)$, with a minor overlap at an MLA of $2.0 \mathrm{~mm}^{2}$. Figure 4 illustrates how quantitative stenosis measurements were performed and Fig. 5 shows an example of a high-grade significant proximal LAD lesion.

\section{Relative $\%$ area and $\%$ diameter stenosis}

The most accurate cut-offs for haemodynamically relevant stenosis by ICA were $74 \%$ area stenosis with a sensitivity of $85 \%(95 \%$ CI 76.1-91.9) and a specificity of $77.9 \%(95 \%$ CI 69.5-84.9). ( $c=0.89 ; 95 \%$ CI 0.84-93).

For diameter stenosis, the optimal threshold was $61 \%$ (c=0.87; $95 \%$ CI 0.82-92).
Table 2 Quantitative coronary computed tomography angiography stenosis parameters

\begin{tabular}{lccc}
\hline & ICA - & ICA & p-value \\
& $\mathrm{n}=84$ & $\mathrm{n}=72$ & \\
\hline Vessels & 86 & 124 & \\
LM & $2(2.6 \%)$ & $8(6.5 \%)$ & n.s. \\
LAD & $47(54.7 \%)$ & $85(68.5 \%)$ & n.s. \\
CX & $24(27.9 \%)$ & $23(18.5 \%)$ & n.s. \\
RCA & $13(15.1 \%)$ & $8(6.5 \%)$ & n.s. \\
Minimal lumen area (MLA, $\left.\mathrm{mm}^{2}\right)$ & $2.61 \pm 0.8$ & $1.34 \pm 0.4$ & $<0.0001 *$ \\
Minimal lumen diameter $(\mathrm{MLD}, \mathrm{mm})$ & $1.44 \pm 2.4$ & $1.0 \pm 0.2$ & 0.13 \\
Diameter stenosis $(\%)$ & $53.4 \pm 6.6$ & $66.1 \pm 8.5$ & $<0.05 *$ \\
Area stenosis ( \%) & $65.6 \pm 9.9$ & $81.4 \pm 7.6$ & $<0.01 *$ \\
Length (mm) & $3.2 \pm 1.8$ & $7.1 \pm 7.8$ & $<0.0001 *$ \\
Calcification at slice of measurement & $54(64.3 \%)$ & $35(48.6 \%)$ & 0.19 \\
Proximal vessel reference area $\left(\mathrm{mm}^{2}\right)$ & & & \\
LAD & $7.8 \pm 2.2$ & $8.6 \pm 3.7$ & 0.182 \\
CX & $6.0 \pm 1.4$ & $5.3 \pm 3.0$ & 0.113 \\
RCA & $7.2 \pm 3.2$ & $9.1 \pm 4.0$ & 0.696 \\
\hline
\end{tabular}

ICA invasive coronary angiography (+positive for high grade stenosis and - negative), n.s. not significant 


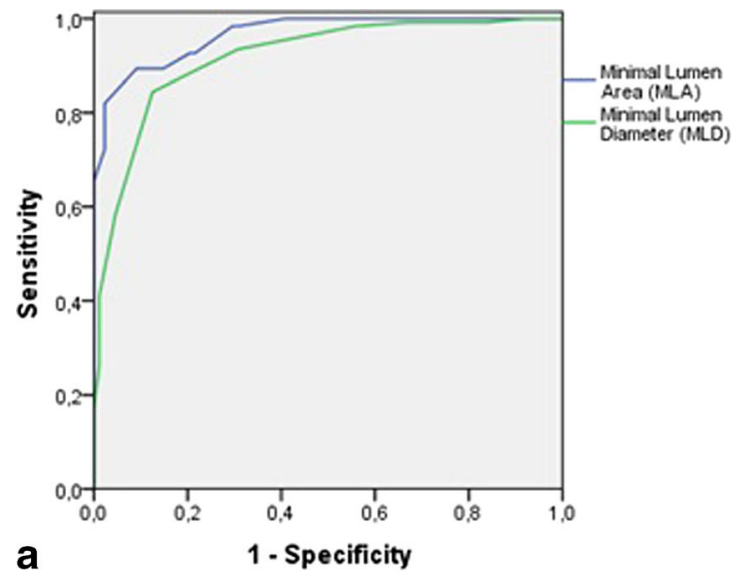

Fig. 1 Receiver operating characteristic (ROC) curves of absolute area and diameter stenosis showed higher accuracy $(c=0.97$ and $c=0.92$, respectively) compared to relative area and diameter stenosis $(\mathrm{c}=0.89$ and $c=0.87$ ) for detection of hemodynamically significant stenosis

\section{Lesion length $(\mathbf{L L})$}

Lesion length showed the lowest accuracy with $\mathrm{c}=0.74$ ( $\mathrm{p}<0.001 ; 95 \%$ CI $0.67-81)$ and an optimal cut-off of $5 \mathrm{~mm}$ (sensitivity $44 \%$ ) while accuracy for the LL/MLA and LL/MLD ratio was higher with $\mathrm{c}=0.904(95 \% \mathrm{CI} 0.86-0.94)$ and $\mathrm{c}=0.84$ $(95 \%$ CI $0.79-0.89)(\mathrm{p}<0.001)$, respectively.

\section{Combined interobserver/manual interactive MPR versus automated cMPR post-processing variability}

Combined interobserver/manual interactive MPR versus automated cMPR post-processing variability was: $\mathrm{MLA} \mathrm{r}=0.63$,

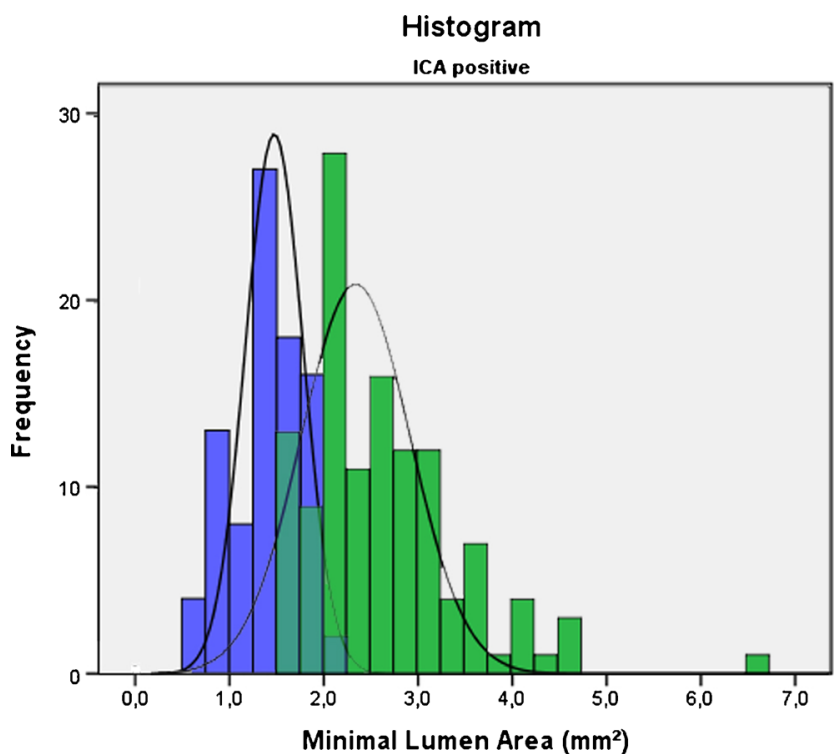

Fig. 2 Absolute minimal lumen area (MLA) by computed tomography angiography (CTA) in heamodynamically significant lesions with subsequent revascularization (invasive coronary angiography positive, blue and negative, green, lesions). All positive lesions had an MLA of less than $2.1 \mathrm{~mm}^{2}$. MLA $\left(\mathrm{mm}^{2}\right)$ is displayed on the $\mathrm{x}$-axis

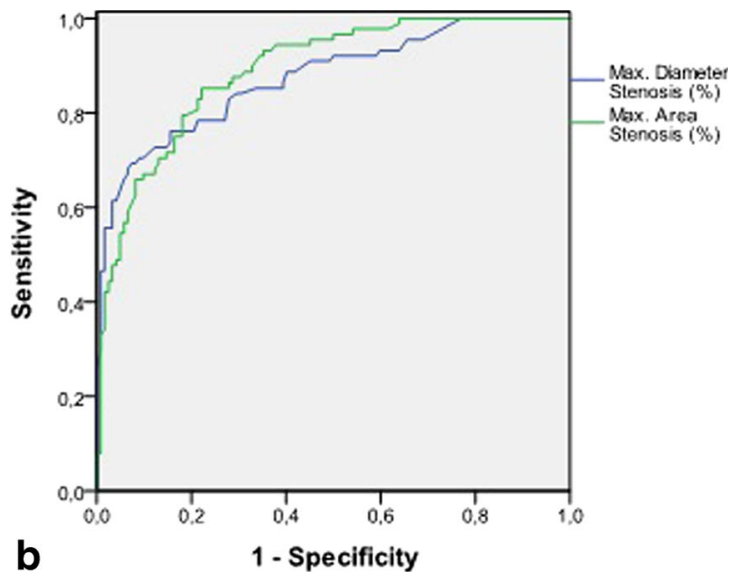

Panel A: Minimal lumen area (MLA) $(c=0.97,95 \%$ CI 0.94-0.99, blue) and minimal lumen diameter (MLD) $(c=0.92$, green) Panel B: Relative \%area ( $c=0.89$, green) and diameter stenosis $(c=0.87$, blue $)$

MLD $r=0.56$, relative \%area stenosis $r=0.4$ and relative $\%$ diameter stenosis $r=0.49(p<0.001)$. Absolute variation was low with 1.9 versus $1.9 \mathrm{~mm}^{2}(\mathrm{p}=0.98)$ for MLA, 1.2 versus $1.3 \mathrm{~mm}(\mathrm{p}=0.007)$ for MLD, $75 \%$ versus $77.2 \%$ for \%area stenosis $(p=0.11)$, while the highest variability was found for \%diameter stenosis with $60 \%$ versus $56 \%$ $(\mathrm{p}=0.005)$.

\section{Discussion}

Our study shows superiority of absolute vessel lumen quantitative parameters (MLA, MLD and LL/MLA ratio) with a higher accuracy for prediction of haemodynamically relevant

Table 3 Minimal lumen area (MLA) by computed tomography angiography for prediction of haemodynamically significant stenosis by invasive coronary angiography. Highest accuracy was found at MLA $1.8 \mathrm{~mm}^{2}$. Values less than $2.1 \mathrm{~mm}^{2}$ were $100 \%$ sensitive for highgrade stenosis

\begin{tabular}{lcclc}
\hline MLA $\left(\mathrm{mm}^{2}\right)$ & Sensitivity $(\%)$ & $95 \% \mathrm{CI}$ & Specificity $(\%)$ & $95 \% \mathrm{CI}$ \\
\hline$<0.4$ & 0.00 & $0.0-4.1$ & 100 & $97.0-100.0$ \\
$\leq 1.4$ & 59.1 & $48.1-69.5$ & 100 & $97.0-100.0$ \\
$\leq 1.5$ & 69.3 & $58.6-78.7$ & 98.4 & $94.2-99.8$ \\
$\leq 1.6$ & 78.4 & $68.4-86.5$ & 92.6 & $86.5-96.6$ \\
$\leq 1.7$ & 85.2 & $76.1-91.9$ & 89.3 & $82.5-94.2$ \\
$\leq 1.8$ & 90.9 & $82.9-96.0$ & 89.3 & $82.5-94.2$ \\
$\leq 1.9$ & 97.7 & $92.0-99.7$ & 82 & $74.0-88.3$ \\
$\leq 2$ & 97.7 & $92.0-99.7$ & 72.1 & $63.3-79.9$ \\
$\leq 2.1$ & 100 & $95.9-100.0$ & 65.6 & $56.4-73.9$ \\
$\leq 6.7$ & 100 & $95.9-100.0$ & 0.00 & $0.0-3.0$ \\
\hline
\end{tabular}

CI confidence interval 
Table 4 Minimal lumen diameter (MLD) by computed tomography angiography for prediction of haemodynamically significant stenosis by invasive coronary angiography. Highest accuracy was found at a threshold of $1.2 \mathrm{~mm}$

\begin{tabular}{lcclc}
\hline MLD (mm) & Sensitivity (\%) & $95 \%$ CI & Sensitivity (\%) & $95 \%$ CI \\
\hline$<0.5$ & 0 & $0.0-4.1$ & 100 & $97.0-100$ \\
$\leq 0.6$ & 9.1 & $4.0-17.1$ & 100 & $97.0-100$ \\
$\leq 0.8$ & 17.1 & $9.9-26.6$ & 99.2 & $95.5-100$ \\
$\leq 0.9$ & 34.1 & $24.3-45.0$ & 99.2 & $95.5-100$ \\
$\leq 1$ & 50 & $39.1-60.9$ & 98.4 & $94.2-99.8$ \\
$\leq 1.1$ & 75 & $64.6-83.6$ & 93.4 & $87.5-97.1$ \\
$\leq 1.2$ & 90.9 & $82.9-96.0$ & 85.25 & $77.7-91$ \\
$\leq 1.3$ & 97.7 & $92.0-99.7$ & 58.2 & $48.9-67.1$ \\
$\leq 1.4$ & 100 & $95.9-100$ & 40.9 & $32.2-50.3$ \\
$\leq 2.3$ & 100 & $95.9-100$ & 0 & $0.0-3$ \\
\hline
\end{tabular}

$C I$ confidence interval

flow-limiting coronary stenosis requiring coronary revascularization, as compared relative \%area and diameter stenosis. The MLA was the most powerful parameter, aside from the MLD and the LL/MLA ratio.

Our MLA cut-off was optimal at $1.8 \mathrm{~mm}^{2}$, which is perfectly in line with Rossi et al. [6] who enrolled a smaller cohort of less than 100 patients. The highest sensitivity was found at
$2.1 \mathrm{~mm}^{2}$ MLA. Accordingly, if MLA ranges within 1.8 and $2.1 \mathrm{~mm}^{2}$, significant stenosis is possible and a myocardial ischaemia test is recommended.

In contrast, Voros et al. [7] reported a higher area cut-off $\left(3.1 \mathrm{~mm}^{2}\right)$ in 85 patients (ATLANTA I and II trials, $27 \%$ ICA positive rate) for CTA, but smaller MLA cut-off values for IVUS $\left(2.6 \mathrm{~mm}^{2}\right)$. This may be explained by a very low prevalence of $\mathrm{LM}$ stenosis $(\mathrm{n}=2,3.8 \%)$ in our study, while Voros et al. [7] did not specify the anatomical vessel segments. The LM has a larger vessel lumen diameter than LAD, RCA and CX, thus higher-cut-off values are likely.

Secondly, absolute lumen diameter (MLD) (cut-off, $1.2 \mathrm{~mm}$ ) was identified as a valuable parameter for prediction of high-grade stenosis by ICA in our study, with slightly lower accuracy and a higher variation, similar to another study (cutoff, $1.5 \mathrm{~mm}$ ) [6]. Absolute diameter sizing is more susceptible to variations than lumen area tracing, because the stenotic vessel lumen is often non-circular and oval-shaped due to a single-side eccentric plaque, thus arbitrary diameter caliper anchor point placement results in higher interobserver variability.

Thirdly, the relative coronary stenosis \% quantification approach was less accurate than absolute parameters: While area $\%$ stenosis matches well with another study [6], our diameter stenosis cut-off was with $61 \%$ higher as compared to other studies (e.g. $48 \%$ [6]). These findings are in line with previous research showing a moderate correlation $(r=0.54)$ for
Fig. 3 Box-plot showed significantly lower minimal lumen area (MLA) in haemodynamically significant stenosis (invasive coronary angiography (ICA) positive) as compared to negatives on ICA $\left(1.34 \mathrm{~mm}^{2}\right.$ versus $2.61 \mathrm{~mm}^{2}$; $\mathrm{p}<0.001$ ), with a minor overlap at the level of a MLA of $2.0 \mathrm{~mm}^{2}$

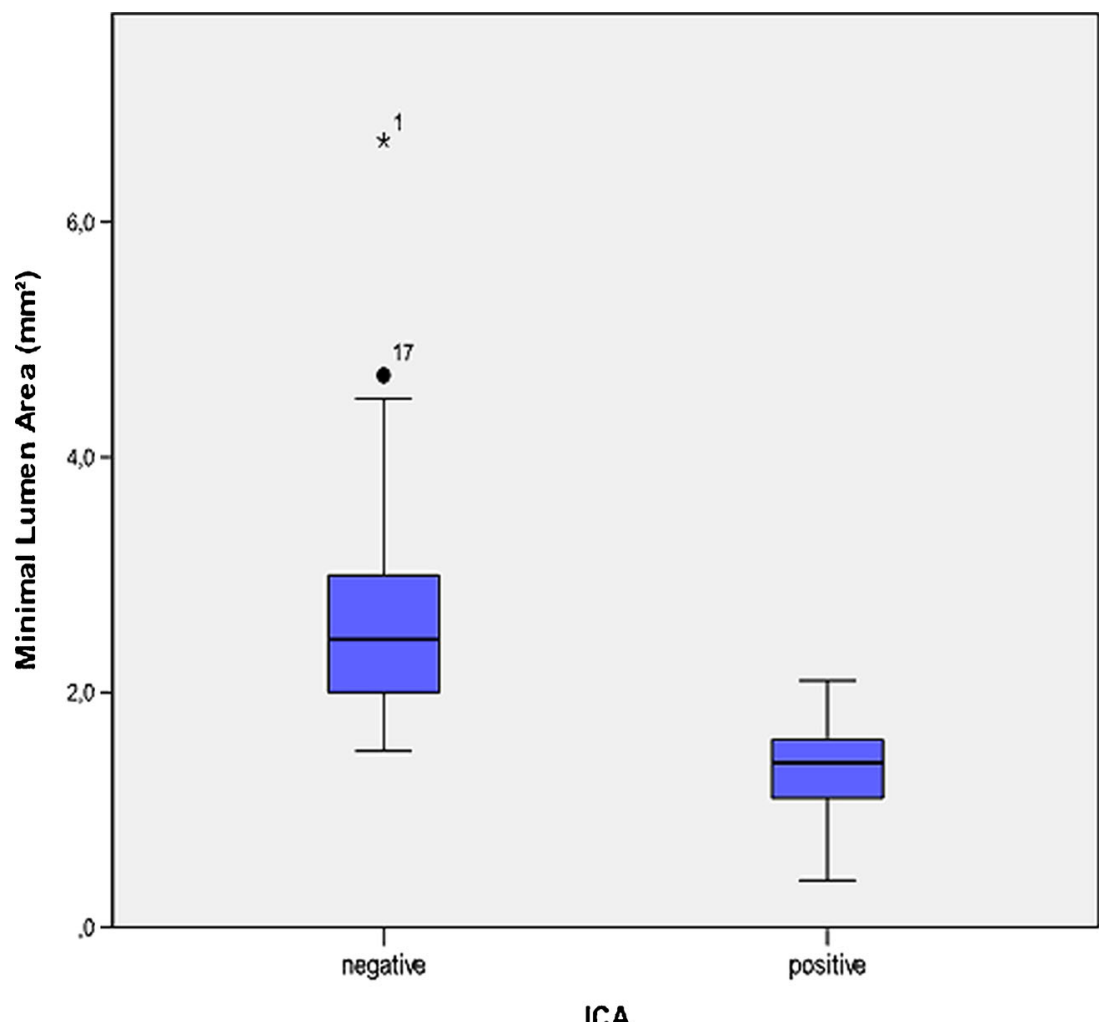

ICA 


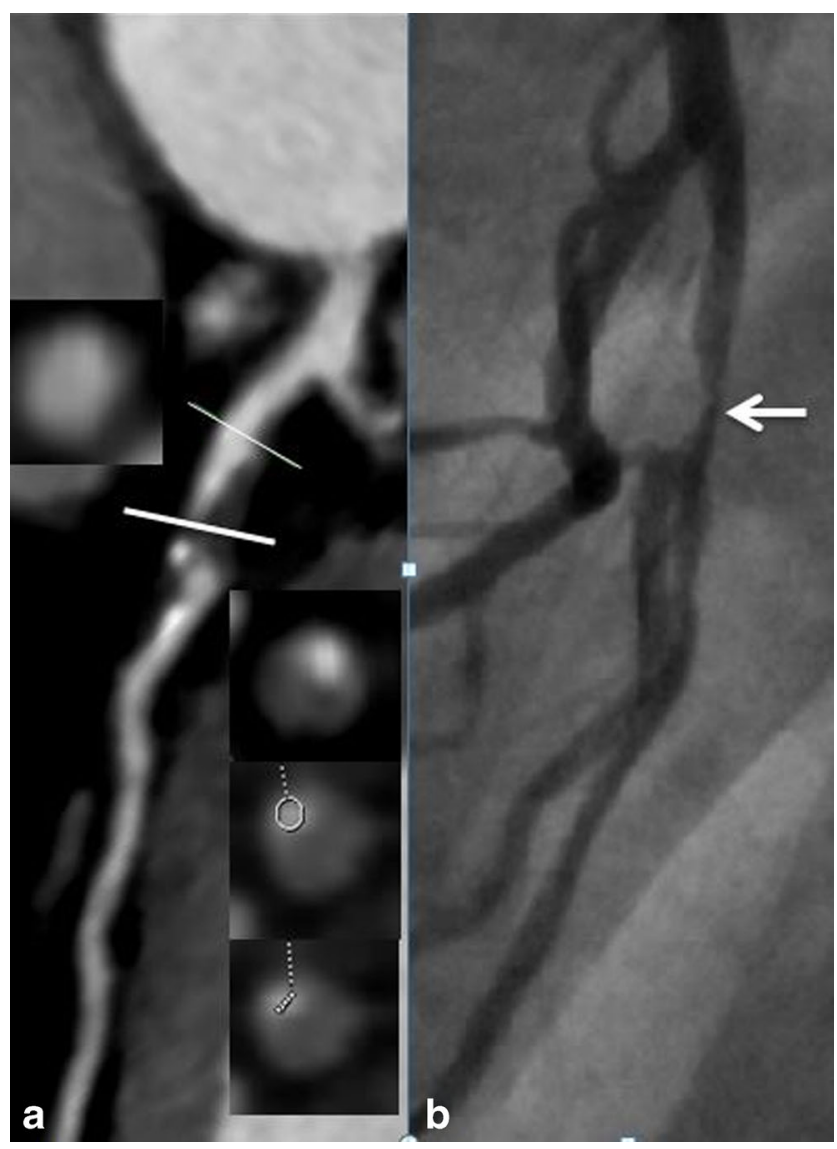

Fig. 4 A 54-year-old male with high-grade LAD stenosis by CT (Panel A, cMPR) with a minimal lumen area of $1.4 \mathrm{~mm}^{2}$ and a diameter of $1.0 \mathrm{~mm}$, as measured on perpendicular cross-sectional images (lower right-sided inlays). The proximal reference vessel area and diameter (upper left inlay, cross-sectional vessel lumen) were measured for calculation of \%area and \% diameter stenosis. Invasive coronary angiography (Panel B) showed $90 \%$ high-grade senosis (white arrow) and percutaneous coronary intervention was performed

correlation of quantitative relative \%stenosis by CTA with ICA [12]. Limited ability for \%stenosis for prediction of haemodynamically significant flow-limiting stenosis meeting the indication for revascularization has been reported previously [13].

Fourthly, LL showed only weak-to-moderate discriminative strength, while LL/MLA and LL/MLD ratios in our study, similar to those of others [14], provided a higher accuracy, implying integration into clinical reporting in practice.

Finally, our results were correlated moderately among multiple observers with different experience levels, who also used two different post-processing techniques (cMPR vs. interactive oblique MRP), enhancing the variation. While the MLA was the most stable parameter with the lowest variation, MLD was highly variable, as discussed above. A variety of factors such as the post-processing technique, image slice selection and individual caliper/area tracer placement influence stenosis quantification results. For primary data analysis, we chose

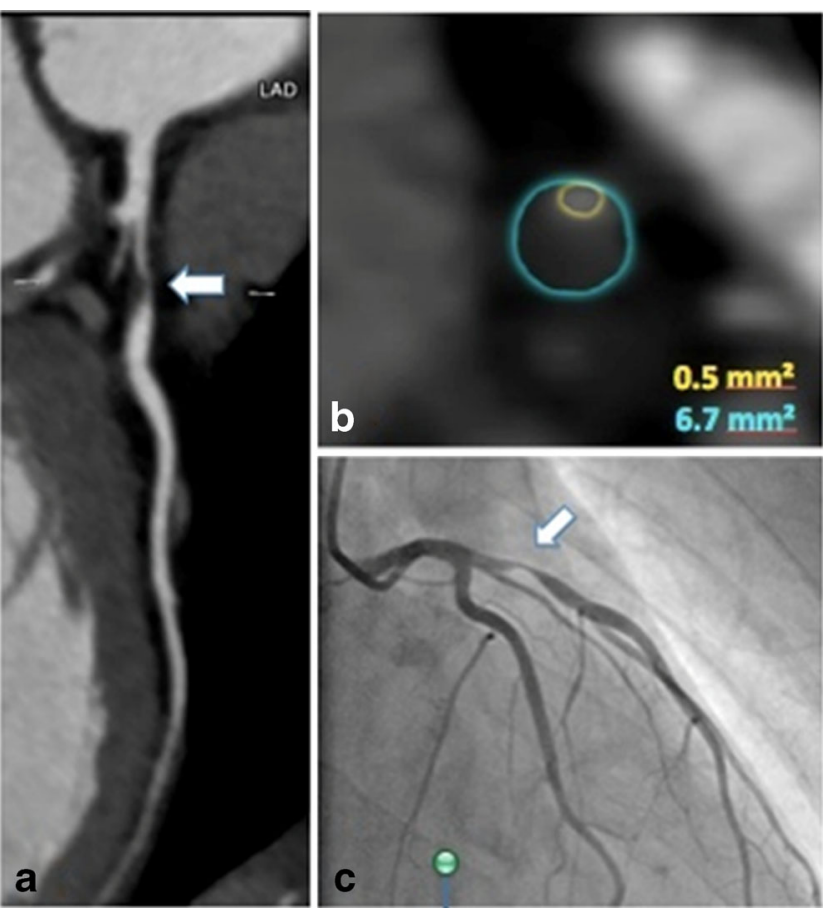

Fig. 5 A 62-year-old-male smoker with a non-calcifying proximal LAD lesion (A). Cross-sectional lumen area (B) was traced with a region of interest (blue) on a proximal reference slice (here, overprojected into the lesion, with a lumen area of $6.7 \mathrm{~mm}^{2}$ (blue)). Minimal lumen area (MLA) of the lesion was $0.5 \mathrm{~mm}^{2}$ (93\% area and $74 \%$ diameter stenosis) (yellow). The lesion was haemodynamically significant on invasive coronary angiography $(\mathbf{C})$ and percutaneous coronary intervention was performed

interactive MPR, which have been validated as the most accurate quantitative technique [15].

\section{Are other CTA parameters useful for prediction of flow-limiting significant stenosis?}

The transluminal attenuation gradient (TAG) downstream of a stenosis assists the estimation of a lesion's functional significance with a lower accuracy of $\mathrm{c}=0.88$ [16] as compared to MLA. A TAG threshold of $\geq 24 \%$ contrast density difference predicted significant lesions with a specificity of $75 \%$ but only a weak sensitivity of $33 \%$ [17].

Furthermore, CT-based noninvasive FFR computation recently showed very promising results (NXT trial) [18, 19], while widespread clinical implementation is currently limited by complex post-processing and only one commercially available type of software.

\section{Which clinical impact has stenosis grading?}

According to the international CONFIRM-registry, estimation of stenosis severity (SSS-score) [20] has powerful prognostic implications for outcome (cardiac event rate). For example, proximal coronary segment with stenosis $>50 \%$ was found to 
be an equivalent high-risk predictor of cardiac events as compared to the risk of smoking [21].

\section{Study limitations}

Firstly, two different CT scanners (64-slice and dual-source 128-slice) were used; however, their spatial resolution was equal and temporal resolution only slightly different, potentially having a minor influence on image quality.

Secondly, only few left main segments were included in our study, thus our data cannot be used for defining significant LM stenosis, while higher absolute lumen area cut-offs are likely.

\section{Study strengths}

Firstly, we report the largest series of $>200$ lesions so far. Secondly, disease prevalence was balanced with $45 \%$ versus $55 \%$ (ICA positive for flow-limiting stenosis vs. ICA negative), and the ratio of intermediate (40-70\%) and severe $(>70 \%)$ lesions by CTA on quantitative analysis was balanced.

Thirdly, inclusion criteria were highy selective with proximal and mid-coronary segments only and a vessel lumen area of $>5 \mathrm{~mm}^{2}$.

Finally, as a reference standard, we used functional flowlimiting lesions defined by ICA (FFR $<0.8$ or $>70 \%$ stenosis and a positive ischaemia test) requiring coronary revascularization.

\section{Conclusions}

Absolute sizing by MLA and MLD are the most powerful parameters for prediction of functional significant flowlimiting coronary stenosis, while LL/MLA ratio and \%stenosis are less accurate though valuable for clinical reporting. Thus quantitative CTA parameters are useful for patient management in terms of assisting the decision-making process of whether to proceed to ICA or not.

MLA $<1.8 \mathrm{~mm}^{2}$ and MLD $<1.2 \mathrm{~mm}$ are most accurate thresholds indicating functionally significant flow-limiting coronary stenosis requiring coronary revascularization.

Subsequently, quantification of coronary stenosis by CTA might further reduce unnecessary diagnostic ICA examinations, through optimizing the accuracy of CTA as a gatekeeper prior to ICA.

Acknowledgements Open access funding provided by University of Innsbruck and Medical University of Innsbruck. The scientific guarantor of this publication is Gudrun Feuchtner. The authors of this manuscript declare no relationships with any companies whose products or services may be related to the subject matter of the article. The authors state that this work has not received any funding. One of the authors has significant statistical expertise (Fabian Plank).
No complex statistical methods were necessary for this paper. Institutional Review Board approval was obtained. Written informed consent was waived by the Institutional Review Board. Approval from the institutional animal care committee was not required because there were no animals included.

No study subjects or cohorts have been previously reported. Methodology: retrospective, diagnostic or prognostic study, performed at one institution.

Open Access This article is distributed under the terms of the Creative Commons Attribution-NonCommercial 4.0 International License (http:// creativecommons.org/licenses/by-nc/4.0/), which permits any noncommercial use, distribution, and reproduction in any medium, provided you give appropriate credit to the original author(s) and the source, provide a link to the Creative Commons license, and indicate if changes were made.

\section{References}

1. Mohareb MM, Qiu F, Cantor WJ et al (2015) Validation of the appropriate use criteria for coronary angiography: a cohort study. Ann Intern Med 162:549-56

2. Douglas PS, Hoffmann U, Patel MR et al (2015) PROMISE investigators. Outcomes of anatomical versus functional testing for coronary artery disease. N Engl J Med 372:1291-300

3. Hou Y, Ma Y, Fan W, Wang Y, Yu M, Vembar M et al (2014) Diagnostic accuracy of low-dose 256-slice multi-detector coronary CT angiography using iterative reconstruction in patients with suspected coronary artery disease. Eur Radiol 24:3-11

4. Park HB, Lee BK, Shin S, Heo R, Arsanjani R, Kitslaar PH et al (2015) Clinical feasibility of 3D automated coronary atherosclerotic plaque quantification algorithm on coronary computed tomography angiography: comparison with intravascular ultrasound. Eur Radiol 25:3073-83

5. Feuchtner G, Loureiro R, Bezerra H et al (2012) Quantification of coronary stenosis by dual source computed tomography in patients: a comparative study with intravascular ultrasound and invasive angiography. Eur J Radiol 81:83-8

6. Rossi A, Papadopoulou SL, Pugliese F et al (2014) Quantitative computed tomographic coronary angiography: does it predict functionally significant coronary stenoses? Circ Cardiovasc Imaging 7: $43-51$

7. Voros S, Rinehart S, Vazquez-Figueroa JG et al (2014) Prospective, head-to-head comparison of quantitative coronary angiography, quantitative computed tomography angiography, and intravascular ultrasound for the prediction of hemodynamic significance in intermediate and severe lesions, using fractional flow reserve as reference standard (from the ATLANTA I and II study). J Am J Cardiol 113:23-9

8. Tonino PA, De Bruyne B, Pijls NH, Siebert U, Ikeno F, van' t Veer $\mathrm{M}$ et al (2009) Fractional flow reserve versus angiography for guiding percutaneous coronary intervention. N Engl J Med 360:213-24

9. Taylor AJ, Cerqueira M, Hodgson JM, Mark D, Min J, O'Gara P et al (2010) ACCF/SCCT/ACR/AHA/ASE/ASNC/NASCI/SCAI/ SCMR 2010 appropriate use criteria for cardiac computed tomography. Circulation 122:e525-5

10. Agatston AS, Janowitz WR, Hildner FJ et al (1990) Quantification of coronary artery calcium using ultrafast computed tomography. J Am Coll Cardiol 15:827-832

11. Austen WG, Edwards JE, Frye RL et al (1975) A reporting system on patients evaluated for coronary artery disease: report of the Ad Hoc committee for grading of coronary artery disease, council on cardiovascular surgery. Am Heart Assoc Circ 51:5-40 
12. Leber AW, Knez A, von Ziegler F, Becker A, Nikolaou K, Paul S et al (2005) Quantification of obstructive and nonobstructive coronary lesions by 64 -slice computed tomography: a comparative study with quantitative coronary angiography and intravascular ultrasound. J Am Coll Cardiol 46:147-54

13. Meijboom WB, Van Mieghem CA, van Pelt N et al (2008) Comprehensive assessment of coronary artery stenoses: computed tomography coronary angiography versus conventional coronary angiography and correlation with fractional flow reserve in patients with stable angina. J Am Coll Cardiol 52:636-43

14. Wang R, Renker M, Schoepf UJ, Wichmann JL, Fuller SR, Rier JD et al (2015) Diagnostic value of quantitative stenosis predictors with coronary $\mathrm{CT}$ angiography compared to invasive fractional flow reserve. Eur J Radiol 84:1509-15

15. Cury RC, Ferencik M, Achenbach S et al (2006) Accuracy of 16slice multi-detector CT to quantify the degree of coronary artery stenosis: assessment of cross-sectional and longitudinal vessel reconstructions. Eur J Radiol 57:345-50

16. Wong DT, Ko BS, Cameron JD et al (2013) Transluminal attenuation gradient in coronary computed tomography angiography is a novel noninvasive approach to the identification of functionally significant coronary artery stenosis: a comparison with fractional flow reserve. J Am Coll Cardiol 61:1271-9

17. Hell MM, Dey D, Marwan M, Achenbach S, Schmid J, Schuhbaeck A (2015) Non-invasive prediction of hemodynamically significant coronary artery stenoses by contrast density difference in coronary CT angiography. Eur J Radiol 84:1502-1508

18. Nørgaard BL, Leipsic J, Gaur S, NXT Trial Study Group et al (2014) Diagnostic performance of noninvasive fractional flow reserve derived from coronary computed tomography angiography in suspected coronary artery disease: the NXT trial (analysis of coronary blood flow using CT angiography: next steps). J Am Coll Cardiol 63:145-55

19. Nørgaard BL, Jensen JM, Leipsic J et al (2014) Fractional flow reserve derived from coronary $\mathrm{CT}$ angiography in stable coronary disease: a new standard in non-invasive testing? Eur Radiol 25: 2282-90

20. Hulten E, Villines TC, Cheezum MK et al (2013) CONFIRM Investigators.Usefulness of coronary computed tomography angiography to predict mortality and myocardial infarction among Caucasian, African and East Asian ethnicities (from the CONFIRM [coronary CT angiography evaluation for clinical outcomes: an international multicenter] registry). Am J Cardiol 111: 479-85

21. Al-Mallah MH, Qureshi W, Lin FY et al (2014) Does coronary CT angiography improve risk stratification over coronary calcium scoring in symptomatic patients with suspected coronary artery disease? Results from the prospective multicenter international CONFIRM registry. Eur Heart J Cardiovasc Imaging $15: 267-74$ 